Relations industrielles

Industrial Relations

\title{
Working Union-Management Relations, par Robert Dubin, Prentice-Hall Inc., Englewood Cliffs, N.J., 1958, 291 pp.
}

\section{Gaston Cholette}

Volume 16, numéro 2, avril 1961

URI : https://id.erudit.org/iderudit/1021815ar

DOI : https://doi.org/10.7202/1021815ar

Aller au sommaire du numéro

Éditeur(s)

Département des relations industrielles de l’Université Laval

ISSN

0034-379X (imprimé)

1703-8138 (numérique)

Découvrir la revue

Citer ce compte rendu

Cholette, G. (1961). Compte rendu de [Working Union-Management Relations, par Robert Dubin, Prentice-Hall Inc., Englewood Cliffs, N.J., 1958, 291 pp.] Relations industrielles / Industrial Relations, 16(2), 271-272.

https://doi.org/10.7202/1021815ar

Tous droits réservés (C Département des relations industrielles de l’Université Laval, 1961
Ce document est protégé par la loi sur le droit d'auteur. L’utilisation des services d'Érudit (y compris la reproduction) est assujettie à sa politique d'utilisation que vous pouvez consulter en ligne.

https://apropos.erudit.org/fr/usagers/politique-dutilisation/ 
Ce volume contient les résultats d'une enquête entreprise par l'Institut de droit comparé de l'Université de Paris, sous la direction de Paul Durand, sur le régime légal des conventions collectives dans les six pays-membres de la Communauté européenne du charbon et de l'acier. Les renseignements qui ont servi à la présente étude sur le fonctionnement du régime dans les différents systèmes juridiques en cause, ont été obtenus par l'intermédiaire d'un spécialiste de la matière dans chaque pays. Des membres d'un groupe de travail de spécialistes du droit du travail auprès de la Haute autorité de la Communauté européenne du charbon et de l'acier ont rempli un questionnaire à cet effet et ont fourni des renseignements complémentaires à l'auteur à l'occasion de contacts personnels.

Le présent ouvrage n'est pas un simple exposé des faits, ni une comparaison purement schématique. Il fait des rapprochements sur chacun des principaux aspects de la législation des pays en présence, mettant ainsi en lumière les ressemblances et les différences qu'elle comporte. Il se termine par des conclusions qui ont été élaborées en commun avec les membres du Groupe de travail de la Haute autorité. Ces conclusions forment une synthèse des comparaisons sur l'ensemble du droit des conventions collectives de travail dans les six pays concernés.

On trouve dans le volume quatre grands chapitres: la conclusion de la convention collective, les effets de la convention collective, l'extension de la convention collective et l'application de la convention collective. Dans le premier chapitre, l'auteur traite des méthodes de conclusion des conventions collectives, du domaine d'application de la convention collective, du contenu de celle-ci et des règles de forme. Sur chacun de ces points, comme d'ailleurs sur tous ceux qui sont étudiés dans les autres chapitres, l'auteur fait des comparaisons entre les six pays.

Le deuxième chapitre, qui porte sur les effets de la convention collective, contient une section sur les principes généraux, une autre sur la partie normative et une troisième sur la partie créatrice d'obligations. Dans la section des principes généraux, l'auteur présente un résumé intéressant des points de vue des pays intéressés sur la nature juridique de la convention collective.

Dans le troisième chapitre, qui traite des conventions collectives extensionnées, il y a une section sur les conventions susceptibles d'extension, une autre sur les procédures d'extension et une troisième sur les effets de l'extension. On trouve dans ce chapitre beaucoup de formules qui peuvent servir à des rapprochements utiles avec le régime des décrets dans la Province de Québec.

Enfin, le dernier chapitre porte sur l'application de la convention collective sans recours aux tribunaux, le contrôle de l'application des conventions collectives par des organismes administratifs et l'application de la convention collective par l'intervention des tribunaux.

On trouve en annexe quelques renseignements statistiques sur les conventions collectives dans les six pays de la Communauté européenne du charbon et de l'acier ainsi qu'une bibliographie limitée aux études de caractère général sur les conventions collectives dans ces pays.

Grâce à la vérification de la documentation et au contrôle exercé sur l'élaboration du plan général de l'ouvrage par l'Institut de droit comparé de l'Université de Paris et le Groupe de travail de spécialistes du droit du travail auprès de la Haute autorité de la Communauté européenne du charbon et de l'acier, le lecteur peut considérer le présent volume comme une source d'information d'une exactitude rigoureuse et comme un modèle d'utilisation de la méthode comparative. L'auteur a traité sa matière en maître. Il ne présente pas un rapport aride et impersonnel mais une étude personnelle fort intéressante.

\section{Gaston Cholette.}

\section{Working Union-Management Relations,} par Robert Dubin, Prentice-Hall Inc., Englewood Cliffs, N.J., 1958, 291 pp.

Cet excellent ouvrage traite, comme son titre l'indique, des relations patronales-ouvrières. Il s'agit d'une étude qui complète celle du même auteur intitulée The World of Work (PrenticeHall Inc., Englewood Cliffs, 1958), celle- 
ci étant consacrée spécialement au monde du travail en dehors des relations patronales-syndicales proprement dites.

L'auteur se propose d'apporter à la littérature traitant des problèmes des relations patronales-ouvrières une contribution originale en envisageant sa matière par le truchement de la sociologie. Il n'y a pas de doute que ce sont les économistes qui se sont intéressés le plus jusqu'à aujourd'hui aux relations industrielles. L'ouvrage de Dubin a le mérite, sinon d'innover tout à fait, du moins celui d'employer l'outil de la sociologie dans un domaine qui en a un si grand besoin.

L'auteur insiste tout spécialement sur la théorie de l'organisation, et sur le processus de la prise des décisions au sein des syndicats ouvriers et des compagnies. Ce processus est évidemment envisagé dans le cas de décisions qui ont trait aux relations entre les deux groupes.

La première partie de l'ouvrage traite de la direction des entreprises et des principaux facteurs qui sont à l'origine des décisions de l'employeur relatives à ses relations avec les groupements ouvriers.

Dans une deuxième partie, l'auteur parle des syndicats ouvriers et des groupements para-syndicaux, de leurs objectifs dans un contexte de rapports de force, enfin des fondements de leurs décisions.

La négociation collective, comme on pouvait s'y attendre, constitue le coeur, le centre de l'ouvrage. L'auteur y consacre beaucoup plus d'espace qu'aux autres questions. Il la considère d'abord en tant que manifestation d'un rapport de forces, puis dans son influence sur le fonctionnement des entreprises. Un autre chapitre porte sur les conflits industriels et les grèves.

Enfin, dans une quatrième partie, l'auteur étudie l'impact des relations patronales-ouvrières sur la société en général, notamment sur la sécurité sociale et le bien-être.

Tous ceux qui sont mêlés aux négociations collectives ou à d'autres activi- tés qui se rattachent aux relations industrielles auraient avantage à lire cet ouvrage.

\section{Gaston Cholette.}

\section{La constitution de la société par actions} en droit comparé, par F. de Sola Canizarès, Les Editions de l'Epargne, Paris, 1959, 173 pp.

Il s'agit du premier volume d'une nouvelle collection, sous le titre de - Publications du Centre français de droit comparé ». Cet ouvrage est en même temps le premier tome d'un grand traité consacré aux sociétés par actions et préparé selon la méthode du droit comparé.

L'auteur commence par analyser la notion actuelle de la société par actions en droit comparé. Il remarque qu'on ne peut pas formuler une définition universelle de la société par actions. Les traits qui semblent essentiels ne sont pas admis dans tous les pays; de plus, les mêmes principes n'ont pas le même sens dans tous les pays. Même le principe de base, c'est-à-dire la responsabilité limitée de l'actionnaire, n'est pas une règle absolue. L'auteur fait un rapide tour d'horizon et en profite pour dégager à la fois les traits communs et les différences dans les législations nationales.

Le deuxième est une sorte de nomenclature des sources légales et la bibliographie sur le sujet. L'auteur classe sa matière selon les pays de Common Law, de l'Europe continentale, de l'Amérique latine, de l'URSS et des républiques populaires, de l'Afrique, de l'Asie et de l'Océanie.

Il aborde ensuite successivement la nature juridique de l'acte constitutif, sa forme et son contenu. Il s'agit ici d'une des parties les plus importantes de la présente étude. L'auteur ne retient que les traits essentiels et ne porte des jugements de valeur qu'avec beaucoup de circonspection et de sobriété.

Les chapitres suivants sont consacrés aux actionnaires, au capital, à la souscription privée et publique, au contrôle et à la publicité de la constitution et à la sanction des irrégularités de la cons- 